\title{
Cross-talk between LRRK2 and PKA: implication for Parkinson's disease?
}

\author{
Elisa Greggio, Luigi Bubacco and Isabella Russo \\ Department of Biology, University of Padova, Via Ugo Bassi 58/B, Padova 35121, Italy \\ Correspondence: Elisa Greggio (elisa.greggio@unipd.it) or Isabella Russo (isabella.russo@unipd.it)
}

Received: 28 0ctober 2016 Revised: 12 December 2016 Accepted: 19 December 2016

Version of Record published: 15 February 2017
Evidence indicates that leucine-rich repeat kinase 2 (LRRK2) controls multiple processes in neurons and glia cells. Deregulated LRRK2 activity due to gene mutation represents the most common cause of autosomal dominant Parkinson's disease (PD). Protein

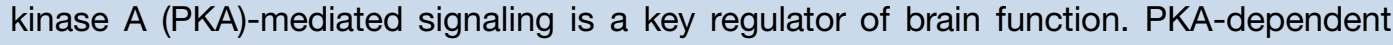
pathways play an important role in brain homeostasis, neuronal development, synaptic plasticity, control of microglia activation and inflammation. On the other hand, a decline of PKA signaling was shown to contribute to the progression of several neurodegenerative diseases, including PD. In this review, we will discuss the accumulating evidence linking PKA and LRRK2 in neuron and microglia functions, and offer an overview of the enigmatic cross-talk between these two kinases with molecular and cellular implications.

\section{Introduction}

Leucine-rich repeat kinase 2 (LRRK2), a gene mutated in autosomal dominant Parkinson's disease (PD) and a risk locus for sporadic PD [1,2], encodes a large and complex protein (Figure 1A) with dual enzymatic function. The presence of serine-threonine kinase and GTPase activities supports a role for LRRK2 in intracellular signaling cascades. Moreover, distinct repeats, including ankyrin, leucine-rich and WD40, present at the N- and C-termini indicate that LRRK2 also acts as scaffold for the assembly of signaling complexes [3]. LRRK2 mutations are clustered within the enzymatic core of the protein, with disease-segregating mutations in the ROC/GTPase (R1441C/H/G and $\mathrm{N} 1437 \mathrm{H}$ ), COR (Y1699C) and kinase (G2019S and I2020T) domains. The observation that all segregating mutations increase LRRK2 cellular activity towards its physiological substrates (LRRK2 itself and Rab proteins) [4,5] and equally impair LRRK2-dependent cellular processes, such as clearance of Golgi-derived vesicles [6], suggests that LRRK2 signaling cascades are probably in the same way affected by mutations. LRRK2 has been linked to distinct signaling cascades, including MAPK [7], Rac/PAK [8,9], Wnt [10], Akt [11], TLR (reviewed in ref. [12]) and cAMP-PKA (protein kinase A) $[13,14]$ pathways. All these pathways can affect multiple cellular processes, but those in which LRRK2 has been convincingly associated are autophagy, cytoskeletal dynamics, membrane trafficking, mitochondria and inflammation (reviewed in ref. [15]). Here we will discuss the emerging evidence placing LRRK2 within the cAMP-PKA signaling pathway and the cellular implications of LRRK2-PKA crosstalk in neurons and microglia cells.

\section{Cellular consequences of PKA-LRRK2 interaction}

PKA (or cAMP-dependent protein kinase) is a tetrameric enzyme composed by a dimeric regulatory subunit (R) (type I or type II) bound to two catalytic subunits (C). Co-operative binding of cAMP molecules to the four binding sites on the $\mathrm{R}$ subunit dimer leads to the dissociation of $\mathrm{C}$ subunits and subsequent activation of enzymatic activity [16]. The tightly regulated spatiotemporal control of PKA activity within specific cellular sites is achieved through interaction with A-kinase anchoring proteins (AKAPs). AKAPs bind the RII subunit dimer and sequester inactive PKA close to its substrates/effectors, thus promoting the phosphorylation of local substrates during the activation state of the enzyme. 


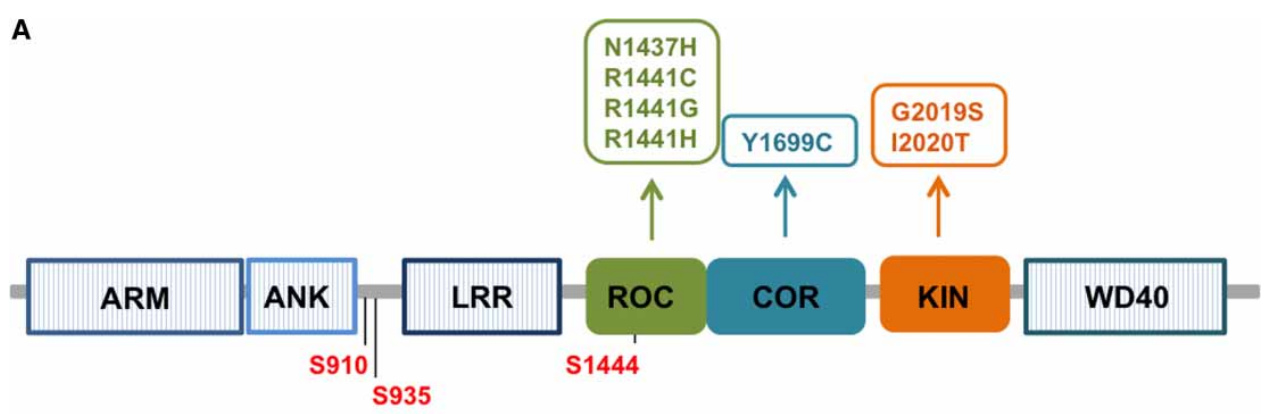

B
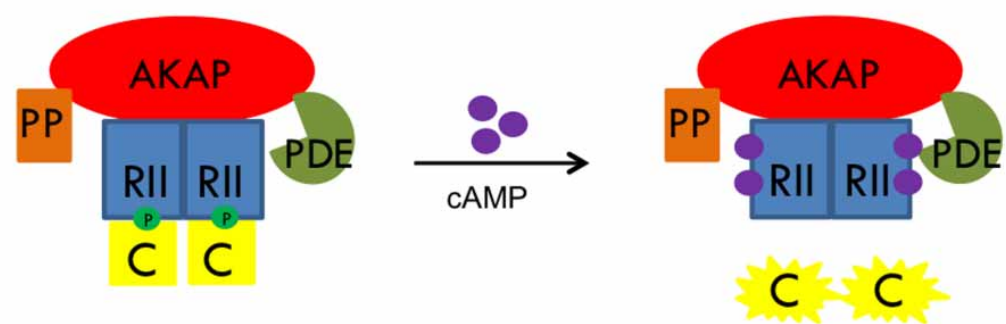

Figure 1. LRRK2 and PKA proteins.

(A) LRRK2 protein contains different domains: Armadillo (ARM), Ankyrin (ANK), leucine-rich repeat (LRR), Ras in complex protein (ROC), C-terminal of Roc (COR), Kinase (KIN) and WD40. S910/S935 between ANK and LRR and S1444 in the ROC domain are phosphorylated by PKA. (B) PKA is a tetrameric enzyme composed by a R dimer (type I or type II) bound to two C subunits. Intracellular increment of cAMP induces the release and activation of $C$ subunits. PKA enzyme is targeted to specific cellular organelles or membranes through interaction with A-kinase anchor proteins (AKAPs). PKA is present in a multifunctional protein complex associated with PP and PDEs.

PKA is part of a multifunctional complex composed of signaling molecules, such as phosphatases (PPs) and phosphodiesterases (PDEs), crucial for signaling compartmentalization and regulation of PKA activation state (Figure 1B) [17]. In neurons, cAMP-PKA signaling is a key regulator of short- and long-term synaptic plasticity (for a comprehensive review, see ref. [18]), whereas in microglia cells it plays an important role as a negative regulator of inflammation [19-21].

In 2014, Parisiadou et al. [13] observed that medium spiny neurons of Lrrk2 knockout mice exhibit abnormal morphology of dendritic spines, which appeared thinner than those of the wild type. Coherently, they found impaired postsynaptic transmission in P15 striatal slices of knockout compared with the wild-type mice. Interestingly, another group did not observe defects in synaptic transmission in acute slices from 3- to 6-month-old Lrrk2 knockout mice, hinting the possibility that LRRK2 activity at dendritic spines is important during the early phases of development [22]. The postsynaptic abnormalities observed by Parisiadou et al. are correlated with an abnormally active PKA with augmented GluR1 and CREB phosphorylation in Lrrk2 knockout mice [13]. It is well established that GluR1 and CREB phosphorylated by PKA are critical for spine maturation and neuron development and plasticity [23,24]. Their results indicate that LRRK2 likely acts upstream of PKA and negatively controls PKA activity, thus modulating neuronal functions. Further supporting a role for LRRK2 upstream of PKA, Lrrk2 knockout neurons also displayed increased cofilin phosphorylation, which affects on actin cytoskeleton dynamics and may explain the spine phenotype observed by the authors [13]. Phosphorylation of cofilin is executed by LIMK1, whose activity is controlled, in part, by PKA phosphorylation [25]. It is interesting to note that we recently reported a link between LRRK2 and LIMK1, which is directly phosphorylated by PAK6, an LRRK2 interactor that promotes actin cytoskeleton remodeling [9]. At the molecular level, LRRK2 interacts with the PKA RII $\beta$ regulatory subunit, and the interaction occurs between LRRK2 GTPase/ROC domain and RII $\beta$-dimerization domain. RII $\beta$-dimerization domain has been shown to be essential for RII-AKAP interaction [26] and deletion of N-terminal 2-5 residues of RII $\beta$ prevents its interaction with LRRK2, suggesting that LRRK2 may operate as AKAP. The engagement of ROC domain in heterologous protein interactions is emerging as a common theme in LRRK2 biology, suggesting that ROC, likely in its 
guanine nucleotide-bound state, is competent to recruit its signaling partners [9,27-31]. Interestingly, immunostaining of endogenous proteins in Lrrk2 knockout neurons reveals mislocalization of RII $\beta$ in dendritic spines compared with wild-type neurons [13]. Together, these observations indicate that LRRK2 regulates PKA activity by controlling PKA subcellular localization acting as dendritic AKAP or AKAP-like. Supporting this idea, neurons derived from mice deficient for MAP2, a well-described dendritic AKAP, also display increased RII $\beta$ localized in dendritic spines and enhanced phosphorylation of GluR1 [32]. In summary, these results suggest that LRRK2 controls PKA activation within dendritic spines, possibly acting as an additional AKAP. Whether LRRK2 controls PKA activity through the modulation of cAMP local concentration by influencing the activity of adenylyl cyclases or PDEs is not known at present. Of note, impaired LRRK2-PKA interaction due to LRRK2 mutation in the ROC domain compromises the signaling at dendritic spines upon dopamine stimulation [13], suggesting that deregulation of this pathway may have pathological relevance.

In a broader contest, deregulation of PKA signaling has been previously linked to PD. Inhibition of cAMP degradation by Rolipram, a PDE4 inhibitor, and overactivation of PKA signaling significantly attenuate 1-methyl-4-phenyl-1,2,3,6-tetrahydropyridine (MPTP)-induced dopamine depletion in the striatum and loss of dopaminergic neurons in the substantia nigra [33]. Moreover, neuronal cells with reduced expression of endogenous PINK1 displayed mitochondrial fragmentation, increased mitochondria-derived superoxide and cellular death, phenotypes that can be rescued by PKA activators or transient expression of AKAP1 that targets endogenous PKA to mitochondria [34]. BE(2)-M17 neuroblastoma cells deficient for PINK1 also exhibited reduction in Drp-1 PKA-mediated phosphorylation, which is important for mitochondrial function [35]. Taken together, these observations suggest that downregulation of cAMP-PKA signaling may affect neuronal functionality at different levels, which may converge into a common pathological outcome.

LRRK2 has been shown to regulate PKA-dependent pathways also in microglia cells. Recently, we demonstrated that primary mouse microglia with Lrrk2 genetic deletion and immortalized BV2 microglia upon LRRK2 pharmacological inhibition exhibit increased PKA-mediated phosphorylation of nuclear NF- $\mathrm{kB}$ p50 subunit [14]. NF- $\mathrm{KB}$ p50:p50 homodimer phosphorylated by PKA is constitutively imported into the nucleus [36], possesses a high affinity for DNA [21] and is essential to repress the transcription of NF- $\mathrm{KB}$ target genes in the absence of extracellular stimuli [37]. Supporting our results, Kim et al. [38] showed that BV2 cells with LRRK2 knockdown display reduced NF- $\kappa \mathrm{B}$ transcription activity and increased p50:p50 homodimer bound to $\mathrm{NF}-\kappa \mathrm{B}$ target sites compared with wild-type cells, further supporting the notion that LRRK2 controls NF- $\mathrm{\kappa B}$

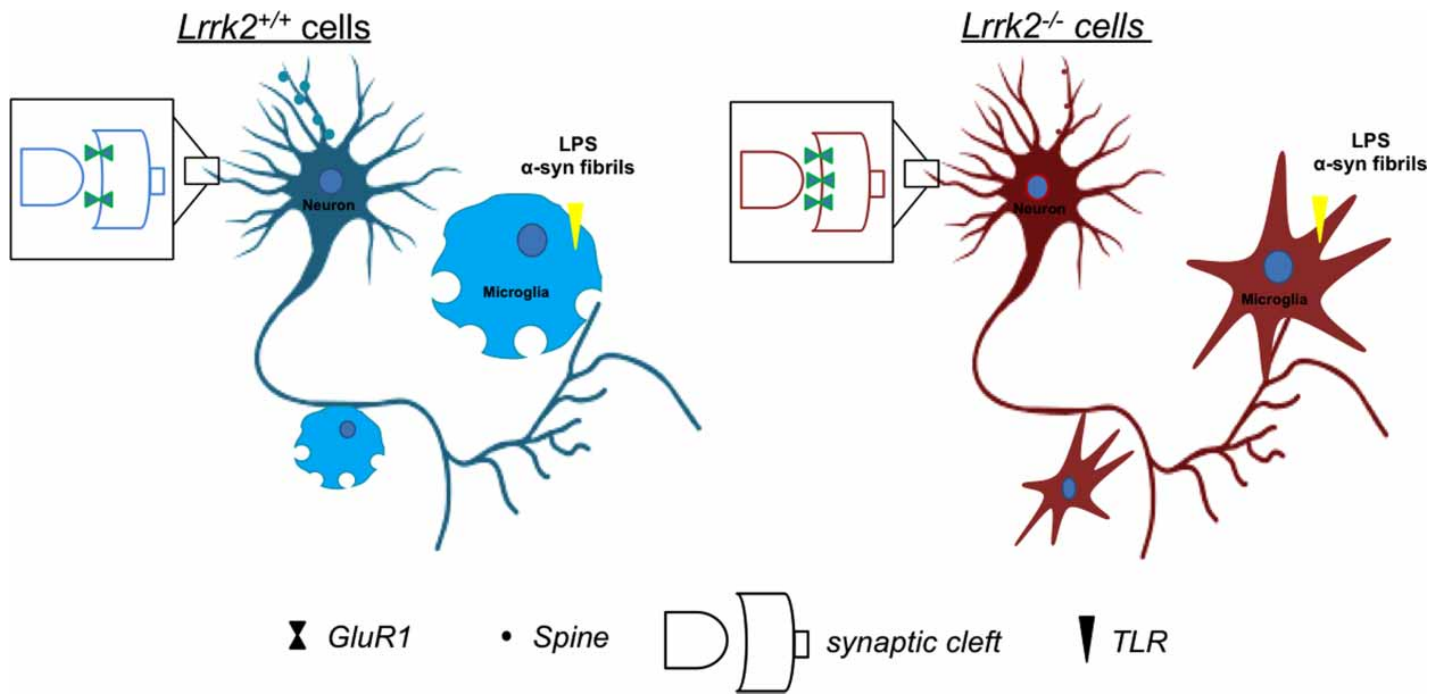

Figure 2. Cellular implication of LRRK2-PKA pathways.

Medium spiny neurons and microglia with $L r r k 2$ genetic deletion exhibit enhanced PKA-dependent signaling. Lrrk2 ${ }^{-/-}$neurons report abnormal synaptic transmission with increased GluR1 phosphorylation and reduced spine area and length compared with $\mathrm{Lrrk}^{+/+}$neurons. $\mathrm{Lrrk}^{-/-}$microglia display reduced activation mediated by $\alpha$-synuclein fibrils or LPS compared with $\mathrm{Lrrk}^{+/+}$cells with increased phosphorylation and binding of $\mathrm{p} 50$ to NF- $\mathrm{kB}$ target sequences. 
p50 inhibitory signaling. Different groups found a reduced inflammation in microglia cells with LRRK2 knockdown or pharmacological inhibition after inflammatory stimuli [14,38-40]. In this regard, we propose that the enhanced phosphorylation of NF- $\mathrm{KB}$ p50 inhibitory subunit bound to DNA might be one molecular mechanism underlying the reduction in NF- $\mathrm{KB}$ target genes transcription upon inflammatory stimuli. An abnormally higher proportion of NF- $\kappa \mathrm{B}$ p50 phosphorylated in the nucleus of LRRK2 knockout or LRRK2-inhibited cells may hamper the binding to DNA of NF- $\mathrm{KB}$ p65:p50 heterodimer and the activation of gene transcription upon inflammatory priming. Based on these findings, we predict that an increase in LRRK2 kinase activity, due to pathological mutations, may attenuate PKA-mediated p50 phosphorylation and its binding to NF- $\mathrm{KB}$ target sites, promoting the binding of active NF- $\mathrm{KB}$ p65:p50 and the transcription of pro-inflammatory mediators. This mechanism may result in increased microglia activation and neuroinflammation with implication for PD. Supporting this hypothesis, LRRK2 G2019S mutation carriers exhibit higher levels of peripheral

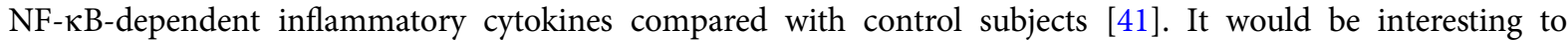
explore the effect of LRRK2 pathological mutations on PKA-mediated NF- $\kappa B$ inhibitory signaling, using, for instance, microglia derived from Lrrk2 G2019S knockin mice. Of note, in contrast from what observed in neurons where LRRK2 regulates PKA activity through localization of RII $\beta$ subunit and independently of its kinase activity, LRRK2 kinase activity is required for PKA function in microglia cells. Future studies directed at understanding whether LRRK2 phosphorylates and/or controls the activity of one of the PKA signaling machinery components will clarify the underlying molecular mechanisms.

\section{Molecular evidence of LRRK2-PKA cross-talk}

Early in 2007, Ito et al. [42] observed that HEK293 cells treated with H-89, a moderately selective but highly potent PKA inhibitor, significantly inhibited the incorporation of phosphate into LRRK2 in a dose-dependent manner. Subsequent studies confirmed that PKA is capable of phosphorylating LRRK2 in vitro and in cells, providing indications on the specific phosphorylation sites [43,44]. Li et al. [43] showed that phosphorylation of S910 and S935 at the N-terminus of LRRK2 is increased upon forskolin (an adenylyl cyclase activator) treatment and is reduced upon PKA pharmacological inhibition. A different study reported that PKA directly phosphorylates LRRK2 S1444 in the ROC domain [44]. It is interesting to note that phosphorylation of both S910/ S935 and S1444 promotes LRRK2 binding to 14-3-3 proteins [43-45], chaperones that are crucial to control the activity of different signaling molecules [46]. Accordingly, multiple lines of evidence indicate that 14-3-3 binding regulates LRRK2 kinase activity [44,47] and/or subcellular localization [48]. In this context, Muda et al. [44] found that LRRK2 R1441C/G/H pathogenic mutations prevent phosphorylation of S1444 by PKA, indicating that a substitution of the nearby arginine at position 1441 may disrupt the PKA recognition motif. Moreover, the authors found that LRRK2 binding to 14-3-3, controlled by PKA phosphorylation at S1444, negatively regulates LRRK2 kinase activity. These results support the notion that PKA controls LRRK2 kinase activity by S1444 phosphorylation and subsequent binding of 14-3-3 proteins, and that the pathogenic effect of ROC mutations may be explained, at least in part, by the downregulation of S1444 phosphorylation. To complicate the scenario, PKA-dependent phosphorylation of S935 also controls LRRK2 binding with 14-3-3 proteins, and the majority of LRRK2 segregating mutations exhibits reduced levels of S935 phosphorylation and impaired binding with 14-3-3s [43,45]. In contrast with S1444, PKA-mediated S935 phosphorylation does not appear to affect LRRK2 kinase activity per se. LRRK2 S910A/S935A immunoprecipitated from MEF (mouse embryonic fibroblasts) cells retains the ability to phosphorylate Rab8 in vitro to a similar extent as LRRK2 wild type [47]. Moreover, LRRK2, dephosphorylated at S910/S935 by pharmacological inhibition, displays similar kinase activity as LRRK2 treated with DMSO [45]. Instead, S910/S935 phosphorylation state profoundly affects LRRK2 cellular localization [49] and, as consequence, the proximity of the kinase to its physiological substrates. Supporting this hypothesis, LRRK2 S910A/S935A knockin MEFs display a dramatic reduction in endogenous Rab10 phosphorylation compared with wildtype cells [47]. The apparent paradox is that the majority of LRRK2 mutations exhibit reduced S935 phosphorylation [45], altered cellular localization [49] but increased kinase activity $[4,5]$. Future studies will hopefully disentangle the precise molecular mechanisms. In addition to PKA, S935 has been reported to be phosphorylated by other kinases, such as CK1 $\alpha$ [50] and IKB kinase family [51], suggesting a multilayer control that can be cell-type or pathway specific. Overall, these observations indicate that phosphorylation of S910/S935 and S1444 may affect LRRK2 catalytic activity, cellular localization and the downstream signaling. Clearly, more investigations are required to clarify the cellular consequences of S910/ S935 and S1444 phosphorylation on LRRK2 pathophysiology. 


\section{Concluding remarks}

Recent literature highlights a functional interaction between LRRK2 and PKA with an enigmatic cross-talk between these two kinases. In the brain, LRRK2 acts upstream of PKA and negatively controls its activity with apparent different mechanisms. In neuronal cells, LRRK2 seems to operate as AKAP to localize PKA signaling at the dendritic spines independently of its kinase activity. Instead, in microglia cells, LRRK2 kinase activity appears to down-regulate PKA signaling through a still unknown mechanism. These observations indicate that LRRK2-dependent regulation of PKA activity and, more in general, LRRK2 physiological function might be cell-type specific. This point seems particularly important, as it may not be appropriate to generalize LRRK2 molecular mechanisms in a cell-type that is not relevant for PD. Understanding whether mutant LRRK2 affects neurons, microglia or astrocytes homeostasis is an exciting future challenge for the field. There is also molecular evidence that PKA acts upstream of LRRK2 through direct phosphorylation. One limitation of these data, however, is that it is not clear whether PKA directly phosphorylates LRRK2 in the cellular context, as these results have been obtained either in vitro or indirectly by increasing cellular cAMP [44,47]. We can speculate that if PKA is upstream of LRRK2, the decline of PKA signaling observed in PD brains may affect LRRK2 binding to 14-3-3 and, as consequence, its kinase activity or its subcellular localization leading to pathological events. Instead, if LRRK2 is upstream of PKA, this may result in an alteration of synaptic transmission in neurons and/or in an exacerbated inflammatory response in microglia when LRRK2 is mutated. Overall, these observations suggest that both directions of LRRK2-PKA interaction might be relevant for the pathogenesis of the disease. Future studies directed at understanding the precise molecular mechanisms underlying LRRK2PKA cross-talk will offer more opportunities to understand the complex LRRK2 pathobiology, with implication for the translation of LRRK2 inhibition strategies into the clinics.

\section{Abbreviations}

AKAP, A-kinase anchoring protein; LRRK2, leucine-rich repeat kinase 2; MEF, mouse embryonic fibroblast; PD, Parkinson's disease; PDE, phosphodiesterase; PKA, protein kinase A; PP, phosphatase; S, serine.

\section{Funding}

We are grateful to the financial support of Michael J. Fox Foundation and InCure (EU Joint ProgrammeNeurogenerative Disease Research, JPND). We also acknowledge Umberto Veronesi Foundation for granting IR with Post-doctoral Fellowship year-2015 and year-2017 Award.

\section{Competing Interests}

The Authors declare that there are no competing interests associated with the manuscript.

\section{References}

1 Paisán-Ruíz, C., Jain, S., Evans, E.W., Gilks, W.P., Simón, J., van der Brug, M. et al. (2004) Cloning of the gene containing mutations that cause PARK8-linked Parkinson's disease. Neuron 44, 595-600 doi:10.1016/j.neuron.2004.10.023

2 Zimprich, A., Biskup, S., Leitner, P., Lichtner, P., Farrer, M., Lincoln, S. et al. (2004) Mutations in LRRK2 cause autosomal-dominant parkinsonism with pleomorphic pathology. Neuron 44, 601-607 doi:10.1016/j.neuron.2004.11.005

3 Marin, I. (2006) The Parkinson disease gene LRRK2: evolutionary and structural insights. Mol. Biol. Evol. 23, 2423-2433 doi:10.1093/molbev/msl114

4 Sheng, Z., Zhang, S., Bustos, D., Kleinheinz, T., Le Pichon, C.E., Dominguez, S.L. et al. (2012) Ser ${ }^{1292}$ autophosphorylation is an indicator of LRRK2 kinase activity and contributes to the cellular effects of PD mutations. Sci. Transl. Med. 4, 164ra161 doi:10.1126/scitransImed.3004485

5 Steger, M., Tonelli, F., Ito, G., Davies, P., Trost, M., Vetter, M. et al. (2016) Phosphoproteomics reveals that Parkinson's disease kinase LRRK2 regulates a subset of Rab GTPases. eLife $\mathbf{5}, 809$ doi:10.7554/eLife.12813

6 Beilina, A., Rudenko, I.N., Kaganovich, A., Civiero, L., Chau, H., Kalia, S.K. et al. (2014) Unbiased screen for interactors of leucine-rich repeat kinase 2 supports a common pathway for sporadic and familial Parkinson disease. Proc. Natl Acad. Sci. U.S.A. 111, 2626-2631 doi:10.1073/pnas.1318306111

7 Chen, C.-Y., Weng, Y.-H., Chien, K.-Y., Lin, K.-J., Yeh, T.-H., Cheng, Y.-P. et al. (2012) (G2019s) LRRK2 activates MKK4-JNK pathway and causes degeneration of SN dopaminergic neurons in a transgenic mouse model of PD. Cell Death Differ. 19, 1623-1633 doi:10.1038/cdd.2012.42

8 Häbig, K., Gellhaar, S., Heim, B., Djuric, V., Giesert, F., Wurst, W. et al. (2013) LRRK2 guides the actin cytoskeleton at growth cones together with ARHGEF7 and Tropomyosin 4. Biochim. Biophys. Acta 1832, 2352-2367 doi:10.1016/j.bbadis.2013.09.009

9 Civiero, L., Cirnaru, M.D., Beilina, A., Rodella, U., Russo, I., Belluzzi, E. et al. (2015) Leucine-rich repeat kinase 2 interacts with p21-activated kinase 6 to control neurite complexity in mammalian brain. J. Neurochem. 135, 1242-1256 doi:10.1111/jnc.13369

10 Sancho, R.M., Law, B.M.H. and Harvey, K. (2009) Mutations in the LRRK2 Roc-COR tandem domain link Parkinson's disease to Wnt signalling pathways. Hum. Mol. Genet. 18, 3955-3968 doi:10.1093/hmg/ddp337

11 Chuang, C.-L., Lu, Y.-N., Wang, H.-C. and Chang, H.-Y. (2014) Genetic dissection reveals that Akt is the critical kinase downstream of LRRK2 to phosphorylate and inhibit FOX01, and promotes neuron survival. Hum. Mol. Genet. 23, 5649-5658 doi:10.1093/hmg/ddu281 
12 Russo, I., Bubacco, L. and Greggio, E. (2014) LRRK2 and neuroinflammation: partners in crime in Parkinson's disease? J. Neuroinflammation 11, 52 doi:10.1186/1742-2094-11-52

13 Parisiadou, L., Yu, J., Sgobio, C., Xie, C., Liu, G., Sun, L. et al. (2014) LRRK2 regulates synaptogenesis and dopamine receptor activation through modulation of PKA activity. Nat. Neurosci. 17, 367-376 doi:10.1038/nn.3636

14 Russo, I., Berti, G., Plotegher, N., Bernardo, G., Filograna, R., Bubacco, L. et al. (2015) Leucine-rich repeat kinase 2 positively regulates inflammation and down-regulates NF-kB p50 signaling in cultured microglia cells. J. Neuroinflammation 12, 230 doi:10.1186/s12974-015-0449-7

15 Wallings, R., Manzoni, C. and Bandopadhyay, R. (2015) Cellular processes associated with LRRK2 function and dysfunction. FEBS J. 282, 2806-2826 doi:10.1111/febs. 13305

16 Taylor, S.S., Buechler, J.A. and Yonemoto, W. (1990) cAMP-dependent protein kinase: framework for a diverse family of regulatory enzymes. Annu. Rev. Biochem. 59, 971-1005 doi:10.1146/annurev.bi.59.070190.004543

17 Dodge-Kafka, K.L., Soughayer, J., Pare, G.C., Carlisle Michel, J.J., Langeberg, L.K., Kapiloff, M.S. et al. (2005) The protein kinase A anchoring protein mAKAP coordinates two integrated cAMP effector pathways. Nature 437, 574-578 doi:10.1038/nature03966

18 Kandel, E.R. (2012) The molecular biology of memory: CAMP, PKA, CRE, CREB-1, CREB-2, and CPEB. Mol. Brain 5, 14 doi:10.1186/1756-6606-5-14

19 Ghosh, M., Aguirre, V., Wai, K., Felfly, H., Dietrich, W.D. and Pearse, D.D. (2015) The interplay between cyclic AMP, MAPK, and NF-KB pathways in response to proinflammatory signals in microglia. BioMed. Res. Int. 2015, 1-18 doi:10.1155/2015/308461

20 Ghosh, M., Xu, Y. and Pearse, D.D. (2016) Cyclic AMP is a key regulator of M1 to M2a phenotypic conversion of microglia in the presence of Th2 cytokines. J. Neuroinflammation 13, 9 doi:10.1186/s12974-015-0463-9

21 Guan, H., Hou, S. and Ricciardi, R.P. (2005) DNA binding of repressor nuclear factor- $\kappa B$ p50/p50 depends on phosphorylation of Ser ${ }^{337}$ by the protein kinase A catalytic subunit. J. Biol. Chem. 280, 9957-9962 doi:10.1074/jbc.M412180200

22 Beccano-Kelly, D.A., Volta, M., Munsie, L.N., Paschall, S.A., Tatarnikov, I., Co, K. et al. (2015) LRRK2 overexpression alters glutamatergic presynaptic plasticity, striatal dopamine tone, postsynaptic signal transduction, motor activity and memory. Hum. Mol. Genet. 24, 1336-1349 doi:10.1093/hmg/ddu543

23 Merz, K., Herold, S. and Lie, D.C. (2011) CREB in adult neurogenesis - master and partner in the development of adult-born neurons? Eur. J. Neurosci. 33, 1078-1086 doi:10.1111/j.1460-9568.2011.07606.x

24 Esteban, J.A., Shi, S.-H., Wilson, C., Nuriya, M., Huganir, R.L. and Malinow, R. (2003) PKA phosphorylation of AMPA receptor subunits controls synaptic trafficking underlying plasticity. Nat. Neurosci. 6, 136-143 doi:10.1038/nn997

25 Nadella, K.S., Saji, M., Jacob, N.K., Pavel, E., Ringel, M.D. and Kirschner, L.S. (2009) Regulation of actin function by protein kinase A-mediated phosphorylation of Limk1. EMBO Rep. 10, 599-605 doi:10.1038/embor.2009.58

26 Burns-Hamuro, L.L., Hamuro, Y., Kim, J.S., Sigala, P., Fayos, R., Stranz, D.D. et al. (2005) Distinct interaction modes of an AKAP bound to two regulatory subunit isoforms of protein kinase A revealed by amide hydrogen/deuterium exchange. Protein Sci. 14, 2982-2992 doi:10.1110/ps.051687305

27 Schreij, A.M., Chaineau, M., Ruan, W., Lin, S., Barker, P.A., Fon, E.A. et al. (2015) LRRK2 localizes to endosomes and interacts with clathrin-light chains to limit Rac1 activation. EMBO Rep. 16, 79-86 doi:10.15252/embr.201438714

28 Chan, D., Citro, A., Cordy, J.M., Shen, G.C. and Wolozin, B.(2011) Rac1 protein rescues neurite retraction caused by G2019S leucine-rich repeat kinase 2 (LRRK2). J. Biol. Chem. 286, 16140-16149 doi:10.1074/jbc.M111.234005

29 Cho, H.J., Yu, J., Xie, C., Rudrabhatla, P., Chen, X., Wu, J. et al. (2014) Leucine-rich repeat kinase 2 regulates Sec16A at ER exit sites to allow ER-Golgi export. EMBO J. 33, 2314-2331 doi:10.15252/embj.201487807

30 Law, B.M., Spain, V.A., Leinster, V.H.L., Chia, R., Beilina, A., Cho, H.J. et al. (2014) A direct interaction between leucine-rich repeat kinase 2 and specific beta-tubulin isoforms regulates tubulin acetylation. J. Biol. Chem. 289, 895-908 doi:10.1074/jbc.M113.507913

31 Athanasopoulos, P.S., Jacob, W., Neumann, S., Kutsch, M., Wolters, D., Tan, E.K. et al. (2016) Identification of protein phosphatase 2A as an interacting protein of leucine-rich repeat kinase 2. Biol. Chem. 397, 541-554 doi:10.1515/hsz-2015-0189

32 Zhong, H., Sia, G.-M., Sato, T.R., Gray, N.W., Mao, T., Khuchua, Z. et al. (2009) Subcellular dynamics of type II PKA in neurons. Neuron 62, 363-374 doi:10.1016/.j.neuron.2009.03.013

33 Yang, L., Calingasan, N.Y., Lorenzo, B.J. and Beal, M.F. (2008) Attenuation of MPTP neurotoxicity by rolipram, a specific inhibitor of phosphodiesterase IV. Exp. Neurol. 211, 311-314 doi:10.1016/i.expneurol.2007.02.010

34 Dagda, R.K., Gusdon, A.M., Pien, I., Strack, S., Green, S., Li, C. et al. (2011) Mitochondrially localized PKA reverses mitochondrial pathology and dysfunction in a cellular model of Parkinson's disease. Cell Death Differ. 18, 1914-1923 doi:10.1038/cdd.2011.74

35 Sandebring, A., Thomas, K.J., Beilina, A., van der Brug, M., Cleland, M.M., Ahmad, R. et al. (2009) Mitochondrial alterations in PINK1 deficient cells are influenced by calcineurin-dependent dephosphorylation of dynamin-related protein 1. PLOS ONE 4, e5701 doi:10.1371/journal.pone.0005701

36 Ten, R.M., Paya, C.V., Israël, N., Le Bail, O., Mattei, M.G., Virelizier, J.L. et al. (1992) The characterization of the promoter of the gene encoding the p50 subunit of NF- $\mathrm{KB}$ indicates that it participates in its own regulation. EMBO J. 11, 195-203 PMID:1740105

37 Hou, S., Guan, H. and Ricciardi, R.P. (2003) Phosphorylation of serine 337 of NF-кB p50 is critical for DNA binding. J. Biol. Chem. 278, 45994-45998 doi:10.1074/jbc.M307971200

38 Kim, B., Yang, M.-S., Choi, D., Kim, J.-H., Kim, H.-S., Seol, W. et al. (2012) Impaired inflammatory responses in murine Lrrk2-knockdown brain microglia. PLOS ONE 7, e34693 doi:10.1371/journal.pone.0034693

39 Marker, D.F., Puccini, J.M., Mockus, T.E., Barbieri, J., Lu, S.-M., Gelbard, H.A. (2012) LRRK2 kinase inhibition prevents pathological microglial phagocytosis in response to HIV-1 Tat protein. J. Neuroinflammation 9, 261 doi:10.1186/1742-2094-9-261

40 Moehle, M.S., Webber, P.J., Tse, T., Sukar, N., Standaert, D.G., DeSilva, T.M. et al. (2012) LRRK2 inhibition attenuates microglial inflammatory responses. J. Neurosci. 32, 1602-1611 doi:10.1523/JNEUROSCI.5601-11.2012

41 Dzamko, N., Rowe, D.B. and Halliday, G.M. (2016) Increased peripheral inflammation in asymptomatic leucine-rich repeat kinase 2 mutation carriers. Mov. Disord. 31, 889-897 doi:10.1002/mds.26529

42 Ito, G., Okai, T., Fujino, G., Takeda, K., Ichijo, H., Katada, T. et al. (2007) GTP binding is essential to the protein kinase activity of LRRK2, a causative gene product for familial Parkinson's disease. Biochemistry 46, 1380-1388 doi:10.1021/bi061960m 
43 Li, X., Wang, Q.J., Pan, N., Lee, S., Zhao, Y., Chait, B.T. et al. (2011) Phosphorylation-dependent 14-3-3 binding to LRRK2 is impaired by common mutations of familial Parkinson's disease. PLOS ONE 6, e17153 doi:10.1371/journal.pone.0017153

44 Muda, K., Bertinetti, D., Gesellchen, F., Hermann, J.S., von Zweydorf, F., Geerlof, A. et al. (2014) Parkinson-related LRRK2 mutation R1441C/G/H impairs PKA phosphorylation of LRRK2 and disrupts its interaction with 14-3-3. Proc. Natl Acad. Sci. U.S.A. 111, E34-E43 doi:10.1073/pnas.1312701111

45 Dzamko, N., Deak, M., Hentati, F., Reith, A.D., Prescott, A.R., Alessi, D.R. et al. (2010) Inhibition of LRRK2 kinase activity leads to dephosphorylation of $\mathrm{Ser}^{910} / \mathrm{Ser}^{935}$, disruption of 14-3-3 binding and altered cytoplasmic localization. Biochem. J. 430, 405-413 doi:10.1042/BJ20100784

46 Dougherty, M.K. and Morrison, D.K. (2004) Unlocking the code of 14-3-3. J. Cell Sci. 117(Pt 10), 1875-1884 doi:10.1242/jcs.01171

47 Ito, G., Katsemonova, K., Tonelli, F., Lis, P., Baptista, M.A.S., Shpiro, N. et al. (2016) Phos-tag analysis of Rab10 phosphorylation by LRRK2: a powerful assay for assessing kinase function and inhibitors. Biochem. J. 473, 2671-2685 doi:10.1042/BCJ20160557

48 Schapansky, J., Nardozzi, J.D., Felizia, F. and LaVoie, M.J. (2014) Membrane recruitment of endogenous LRRK2 precedes its potent regulation of autophagy. Hum. Mol. Genet. 23, 4201-4214 doi:10.1093/hmg/ddu138

49 Lobbestael, E., Zhao, J., Rudenko, I.N., Beylina, A., Gao, F., Wetter, J. et al. (2013) Identification of protein phosphatase 1 as a regulator of the LRRK2 phosphorylation cycle. Biochem. J. 456, 119-128 doi:10.1042/BJ20121772

50 Chia, R., Haddock, S., Beilina, A., Rudenko, I.N., Mamais, A., Kaganovich, A. et al. (2014) Phosphorylation of LRRK2 by casein kinase $1 \alpha$ regulates trans-Golgi clustering via differential interaction with ARHGEF7. Nat. Commun. 5, 5-827 doi:10.1038/ncomms6827

51 Dzamko, N., Inesta-Vaquera, F., Zhang, J., Xie, C., Cai, H., Arthur, S. et al. (2012) The IкB kinase family phosphorylates the Parkinson's disease kinase LRRK2 at Ser935 and Ser910 during toll-like receptor signaling. PLOS ONE 7, e39132 doi:10.1371/journal.pone.0039132 